\title{
Emergency Drug Procurement using Data Mining
}

\author{
Vishal Nehete \\ Dept. of Information Technology \\ D.Y Patil Institute of Engineering and Technology, \\ Ambi Pune, India \\ Aditi Mahale \\ Dept. of Information Technology \\ D.Y Patil Institute of Engineering and Technology, \\ Ambi Pune, India
}

\author{
Hitesh Prajapati \\ Dept. of Information Technology \\ D.Y Patil Institute of Engineering and Technology, \\ Ambi Pune, India \\ Simran Pandita \\ Dept. of Information Technology \\ D.Y Patil Institute of Engineering and Technology, \\ Ambi Pune, India
}

\author{
Shruti Chaudhari \\ Assistant Professor, \\ Dept. of Information Technology \\ D.Y Patil Institute of Engineering and technology, \\ Ambi Pune, India
}

\begin{abstract}
Unfavourable medication occasions remain a main clarification for dismalness and mortality round the world. Numerous unfavourable occasions aren't distinguished all through clinical preliminaries before a medication gets endorsement to be utilized inside the center. Luckily, as a piece of post selling police examination, regulative organizations and elective foundations keep up mammoth accumulations of unfriendly occasion reports, and these databases blessing an opportunity to survey medication impacts from patient populace information. However, unsupportive components like associative prescriptions, quiet socioeconomics, tolerant restorative narratives, and purposes behind recommending a medication ordinarily square measure uncharacterized in unconstrained inclusion frameworks, and these oversights will confine the work of quantitative gathering ways utilized in the examination of such data. Here, we present a versatile huge information driven methodology for remedying these variables in cases for which the covariates are obscure or unmeasured and consolidate this methodology with existing strategies to improve examinations of medication impacts utilizing three test information sets. Shows the close by medicals to client any place that prescriptions are accessible.
\end{abstract}

Keywords-Big-data, Drug procurement planning, Morbidity prediction, Deep learning, Naive Bayes Algorithm, Haversine Algorithm.

\section{INTRODUCTION}

As several aspects of healthcare service delivery, like finding a doctor or hospital, booking a meeting, paying fees, booking lab tests, viewing lab test reports, have become online, pharmacy is a place that is still lagging behind. Ideally, it's natural to gradually shift from conventional brick-and-mortar pharmacies to on-line ones, but the explanations we still haven't got there are: threat to conventional pharmacies and inefficiencies in monitoring and control [5].

So, our system proposes methodology therefore in associate economical and effective method together with following the normal approach of progressing to medical store search to urge the medication and conjointly verifying it first whether or not the medication is offered there or not by using an android application put in on user's smartphone [6].

Dataset of this module contains various restorative stores' information [6]. Nobody however Admin can approach this. Head adds the restorative information to database and at whatever point customer glance through system demonstrates him near to stores [5]. Exactly when customer enters reactions.

- System separate that signs with colossal data.

- Detect the ailment or affectability he is encountering.

- Recommend reasonable drugs to fix that affliction.

- For doing this we are using a Naive Bayes classifier [3] which will analyse the symptoms given as an input to the system by user, and can counsel a correct and appropriate medication to the user.

- Shows the near by medicals wherever that medications are available.

We will be using Haversine algorithmic rule [2] to perform the above task,

- Which can counsel user the closest medical store which can have that specific medication.

\section{LITERATURE SURVEY}

A systematic review of literatures on public health, the importance of pharmaceutical supply management in public health, strategic and design issues in the current public pharmaceutical supply chain and the need of information technology applications from the logistics perspective to gain supply chain visibility, which results in provision of enhanced healthcare services [10]. Papers and reports submitted by World Health Organizations, peer-reviewed academic work papers and journals were gathered for content analysis. Scientific search engines with bibliographic databases were searched to gather information related to the area of interest. None-relevant articles were excluded by 
outcome of interest and the researcher's boundaries of study. The process of literature review for this research is highly context specific and focusses on the pharmaceutical purchasing and distribution for public healthcare system in a mid-income state, Karnataka, India.

Naive Bayes is the popular algorithm for classification based on Bayes Theorem. Bayes Theorem describe the probability based on previous knowledge. Naive Bayes model is a relatively easy model of classification, this model assumes that all the attributes are independent given the value of class variables [3]. Based on the features, the algorithms aim is to evaluate the label of unknown labelled objects. The issue with the Naive Bayes classifier is that it assumes that all attributes are independent, in real world problem this assumption gets violated. Thus there arrived a need to extend the Naive Bayes algorithm in order to handle the dependencies between the attributes.

Classification is the predictive technique of the category to which the data belongs. It is a technique of predicting the class of an instance from a set of attributes describing that instance. Naive Bayes model is a relatively easy model of classification, this model assumes that all the attributes are independent given the value of class variables. This is not a single algorithm but a group of algorithms which consists of three classifiers. In a classification problem, a set of labelled training set is given to the algorithm, these are defined by some features. Based on the features, the algorithms aim is to evaluate the label of unknown labelled objects. In a at classification problem, $\mathrm{c}$ an element of $\mathrm{C}$ is will get assigned to every test example. This will output only a single class for every test example. One more issue with the Naive Bayes classifier is that it assumes that all attributes are independent, in real world problem this assumption gets violated. Thus there arrived a need to extend the Naive Bayes algorithm in order to handle the dependencies between the attributes.

Haversine is the distance identification algorithm in given spherical trigonometry. The haversine formula is an equation important in navigation, giving great circle distances between two points on a sphere from their longitudes and latitudes [1]. It is a special case of a more general formula in spherical trigonometry, the law of haversines, relating the sides and angles of spherical triangles [4].

Location based services (LBS) is one of the new technologies to gain a position in the geographic presence of mobile devices [4]. The presence of the LBS technique can be used for information mapping of an area such as the existence of an event. The existence of the event is inuenced by the number of visitor to the event, as it may attract people to join the event. Haversine formula is the method used to calculate the distance from two different points. In this study, the Haversine formula applied to calculate the distance between users with a set of events [1]. To determine the presence of visitors is done by calculating the results of haversine formula with a radius field of each of these events. From the calculations, if the users are in the radius area, the number of visitors the event will be calculated and updated. In this research, we applied this method in android application. Therefore, the haversine formula can be used to calculate the distances and can be implemented to determine the number of visitors who signed into events[1][4].

\section{ALGORITHMS}

\section{A. Haversine Algoritm}

Haversine Formula is utilized to figure geographic separation on earth. On the off chance that you have two distinctive scope-longitude estimations of two diverse point on earth, at that point with the assistance of Haversine Formula, you can without much of a stretch register the extraordinary circle separation (The most brief separation between two on the outside of a Sphere). The term Haversine was begat by Prof. James Inman in 1835 .

Haversine recipe can be determined as:

Focal edge Haversine can be figured, between two with $r$ as range of earth, $d$ as the separation between two, is scope of two points and is longitude of two points respectively as:

$$
d=2 r \arcsin \sqrt{\sin ^{2} \frac{\left(X_{0}-X_{0}\right)}{2}+\cos \left(X_{1}\right) \cos \left(X_{2}\right) \sin ^{2} \frac{(\theta)}{2}}
$$

\section{B. Nä̈ve Bayes Algorithm}

Naive Bayes is a probabilistic algorithm that can be used in a wide variety of classification tasks [3]. Typical applications include filtering spam, classifying documents,sentiment prediction etc. The name naive is used because it assumes the features that go into the model is independent of each other. That is changing the value of one feature, does not directly influence or change the value of any of the other features used in the algorithm. This algorithm is popular because there is a significant advantage with NB. Since it is a probabilistic model, the algorithm can be coded up easily and the predictions made real quick. Real-time quick. Because of this, it is easily scalable and is traditionally the algorithm of choice for real-world applications (apps) that are required to respond to user's requests instantaneously.

\section{- Bayes Theorem}

The Bayes Rule is a way of going from $\mathrm{P}(\mathrm{X} \mid \mathrm{Y})$, known from the training dataset, to find $\mathrm{P}(\mathrm{Y} \mid \mathrm{X})$. To do this, we replace $\mathrm{A}$ and $\mathrm{B}$ in the above formula, with the feature $\mathrm{X}$ and response $\mathrm{Y}$. For observations in test or scoring data, the $\mathrm{X}$ would be known while $\mathrm{Y}$ is unknown. And for each row of the test dataset, you want to compute the probability of $\mathrm{Y}$ given the $\mathrm{X}$ has already happened [3][7].

$$
\mathrm{p}(\mathrm{A} / \mathrm{B})=\frac{\mathrm{p}\left(\frac{B}{\mathrm{M}}\right) \mathrm{p}(\mathrm{A})}{\mathrm{p}(\boldsymbol{B})}
$$

\section{- The Naive Bayes}

The Bayes Rule provides the formula for the probability of Y given X. But, in real world problems, you typically 
have multiple $\mathrm{X}$ variables [3]. When the features are independent, we can extend the Bayes Rule to what is called Naive Bayes.It is called 'Naive' because of the naive assumption that the X's are independent of each other. Regardless of its name, it's a powerful formula [3].

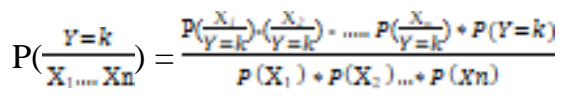

\section{PROPOSED SYSTEM}

We propose a new system for drug planning with the aim of maximizing the expected therapeutic effect on the predicted cases [2]. The combination of the algorithm provide a novel and effective way to support medical preparation for disease control. This system coordinates with all the activities related to procurement of medicines. When the user enters the symptoms, this system will analyse those symptoms properly and after that displays with the relevant medicines to the user as the output [9]. Moreover, it also focuses on the medical store in which the medicines would be available and provides the user with the nearest of all the medical store according to the user's location with the help of GPS system in his mobile [8]. This would help the user in the proper knowledge of the medicines as well as the proper location of the nearest medical store which of course saves user's time and money [2].

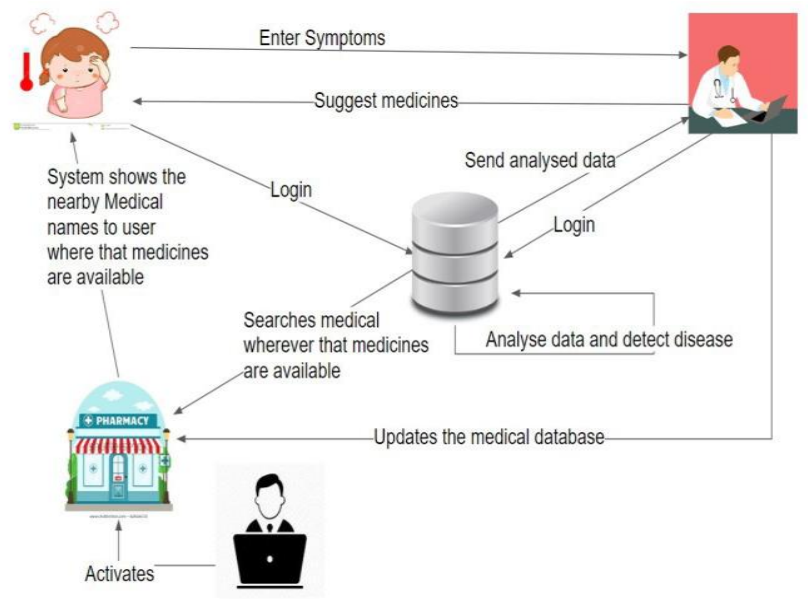

Figure 1:System Architecture

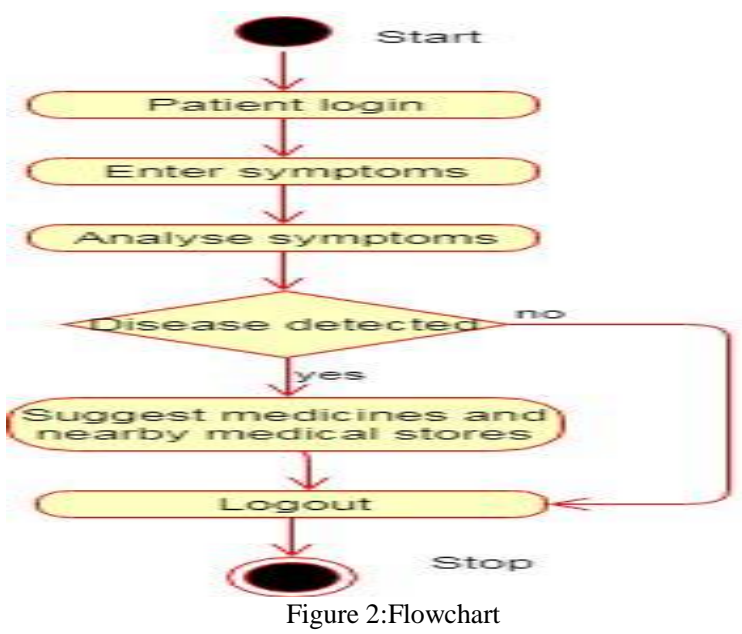

V. CONCLUSIONThis paper put forward a big-data driven approach to predict the diseases and then enable to produce an emergency drug procurement plan to prepare for the upcoming cases. Through latest big data technology, the identification and monitoring of diseases have become easy and accurate compare to traditional methods. This paper also shows that there is drastic rise in accuracy and minimization in error rate compare to previous technology. After studying different algorithms we came to a conclusion that Naive Bayes algorithm gives better classification result and to determine the spherical distance Haversine is the best algorithm. Therefore, these algorithms will help to reduce the error rate and also increase the accuracy of the application.

\section{REFERENCES}

[1] Ronak Chavan and Manish Rana, "A literature review on hierarchical Naive Bayes classifier," ISSN: 2321-9653; IC Value: 45.98; SJ Impact Factor: 6.887, Volume 7 Issue IV, Apr 2019.

[2] Mykhailo Granik and Volodymyr Mesyura, "Fake news detection using Naive Bayes classifier," INSPEC Accession Number: 17353859, DOI: 10.1109/UKRCON.2017.8100379, Publisher: IEEE, 2017.

[3] Prof. Nitin R.Chopde and Mr. Mangesh K. Nichat, "Landmark based shortest path detection by using $\mathrm{A}^{*}$ and Haversine formula," ISSN (Online): 2320 - 9801, Vol. 1, Issue 2, April 2013.

[4] Cecep Nurul Alam, Khaerul Manaf, Aldy Rialdy Atmadja, Digital Khrisna Aurum," Implementation of Haversine formula for counting event visitor in the radius based on android application," INSPEC Accession Number: 16340518, DOI: 10.1109/CITSM.2016.7577575, Publisher: IEEE, April 2016.

[5] J. D. Quick, "Applying management science in developing countries: ABC analysis to plan public drug procurement," SocioEco. Plan. Sci., vol. 16, no. 1, pp. 39-50, 1982.

[6] M. Duggan and F. M. Scott Morton, "The distortionary effects of government procurement: Evidence from Medicaid prescription drug purchasing," Quar. J. Eco., vol. 121, no. 1, pp. 1-30, 2006.

[7] Y. Shu and R. Tong, "Common problems of hospital drugs procurement and its solutions," China Pharm., vol. 22, no. 33, pp. 3114-3115, 2011.

[8] H. Bibi, A. Nutman, D. Shoseyov, M. Shalom, R. Peled, S. Kivity, and J. Nutman, "Prediction of emergency department visits for respiratory symptoms using an artificial neural network," Chest, vol. 122 , no. 5, pp. 1627-1632, 2002.

[9] J. Gong and J. Li, "Drug demand combination prediction based on neural networks,” China Manag. Inform. , vol. 17, no.8, pp. 84-88, 2014.

[10] M. S. Sohn, "Demand forecasting for developing drug inventory control model in a university hospital," Korean Journal of Preventive Medicine, vol. 16, no. 1, pp. 113-120, 1983. 